\title{
Use of female information in dairy cattle genomic breeding programs
}

\author{
N. Mc Hugh, ${ }^{*} \dagger^{1}$ T. H. E. Meuwissen, $\ddagger$ A. R. Cromie, $\S$ and A. K. Sonesson\# \\ *Animal and Bioscience Research Department, Animal and Grassland Research and Innovation Centre, Teagasc, Moorepark, Fermoy, Co. Cork, \\ Ireland \\ †School of Agriculture, Food Science and Veterinary Medicine, University College Dublin, Ireland \\ łUniversity of Life Sciences, PO Box 5003, 1432 Ås, Norway \\ §lrish Cattle Breeding Federation, Highfield House, Bandon, Co. Cork, Ireland \\ \#Nofima Marine, PO Box 5010, 1432 Ås, Norway
}

\section{ABSTRACT}

Genomic selection has the potential to increase the accuracy of selection and, therefore, genetic gain, as well as reducing the rate of inbreeding, yet few studies have evaluated the potential benefit of the contribution of females in genomic selection programs. The objective of this study was to determine the effect on genetic gain, accuracy of selection, generation interval, and inbreeding, of including female genotypes in a genomic selection breeding program. A population of approximately 3,500 females and 500 males born annually was simulated and split into an elite and commercial tier representation of the Irish national herd. Several alternative breeding schemes were evaluated to quantify the potential benefit of female genomic information within dairy breeding schemes. Results showed that the inclusion of female phenotypic and genomic information can lead to a 3-fold increase in the rate of genetic gain compared with a traditional BLUP breeding program and decrease the generation interval of the males by 3.8 yr, while maintaining a reasonable rate of inbreeding. The accuracy of the selected males was increased by $73 \%$ in the final $3 \mathrm{yr}$ of the genomic schemes compared with the traditional BLUP scheme. The results of this study have several implications for national breeding schemes. Although an investment in genotyping a large population of animals is required, these costs can be offset by the greater genetic gain achievable through the increased accuracy of selection and decreased generation intervals associated with genomic selection.

Key words: genomic selection, genotyped cow, genetic progress

\footnotetext{
Received November 18, 2010.

Accepted March 31, 2011.

${ }^{1}$ Corresponding author: noirin.mchugh@teagasc.ie
}

\section{INTRODUCTION}

Genomic selection has been described as the most promising application of molecular genetics in livestock populations since work began almost $20 \mathrm{yr}$ ago (Sellner et al., 2007). It is based on the use of dense markers, which are spread across the genome, and whose effects are estimated and used for the prediction of breeding values. The generation of direct genomic value (DGV) involves genotyping a large number of animals with phenotypic information to facilitate the estimation of marker effects and using these marker effects to predict the DGV of genotyped animals with no phenotypes (Schaeffer, 2006; Sellner et al., 2007; Hayes et al., 2009).

Previous simulation studies showed that genomic breeding programs have the potential to increase the accuracy of selection and, therefore, genetic gain (Schaeffer, 2006; König et al., 2009; Lillehammer et al., 2010), as well as decreasing the rate of inbreeding compared with conventional selection methods (Daetwyler et al., 2007; König et al., 2009). The introduction of genomic selection may eliminate or decrease the necessity for structured progeny testing of bulls, thereby leading to savings for the respective industries (Schaeffer, 2006).

Internationally, progeny-tested bulls are being used to estimate marker effects (Berry et al., 2009; VanRaden et al., 2009; Harris and Johnson, 2010), but in the future, the continual emergence of new proven bulls may be limited because of the uptake of genomic selection. Therefore, the potential use of females, with their own performance records, to estimate marker effects becomes increasingly important, especially in countries with small populations. This is a particularly pertinent question, as the cost of genotyping is likely to decrease considerably, either through advancements in genotyping technology or the development of smaller, less dense marker panels coupled with imputation algorithms (Weigel et al., 2010; Zhang and Druet, 2010), or both. Few studies have evaluated the potential benefit of the contribution of females in genomic selection programs (Schaeffer, 2006; Buch et al., 2010; Pryce et al., 2010). 
Females can both be included in the training set or selected on their DGV. Also a decrease in generation interval in the dam-bull pathway can contribute significantly to genetic gain within the population as a whole.

The objective of this study, therefore, was to determine the effect on genetic gain, accuracy of selection, generation interval, and inbreeding of including female genotypes in a genomic selection breeding program. Several schemes, with varying numbers of females selected and genotyped, were investigated to quantify the potential of including female information.

\section{MATERIALS AND METHODS}

A simulation program described previously by Sonesson and Meuwissen (2009) was used in this study.

\section{Population Structure}

The effective population size $\left(\mathrm{N}_{\mathrm{e}}\right)$ in the present study consisted of 200 unrelated animals (100 males and 100 females). This population structure was iterated for 4,000 generations in accordance with the FisherWright population model (Fisher, 1930; Wright, 1931; i.e., animals were randomly selected and mated using sampling with replacement within each generation). In generation 4,000, a base population (Gen0) was created by increasing the number of animals to 3,000 (375 males and 2,625 females) by using more matings (i.e., dams could be chosen more than once for mating). These animals were genotyped to estimate marker effects (described later). To allow for the comparison between the traditional breeding schemes and genomic selection breeding schemes, all animals (males and females) within the base population (year zero) were assumed to be progeny tested with 100 offspring each and, therefore, had highly accurate EBV; these animals were potential selection candidates until 6 yr of age.

After random mating within the base population, Gen1 was produced and had a gender population structure as in Gen0. Animals from Gen1 on were potential selection candidates. Animals eligible for selection were split into 2 tiers, reflecting the fragmentation of the Irish dairy industry (Figure 1). For computation reasons, the numbers of animals in Figure 1 are smaller than those of the actual Irish dairy cattle population, but the selected fractions are similar to the actual fractions. The first tier (elite tier), referred to as the nucleus selected population, represented the elite animals born out of elite matings; a population of commercial animals (i.e., commercial tier) were born out of commercial matings. A population of approximately 3,500 females and 500 males born annually were simulated from Gen1 onwards (Figure 1). Although the size of the entire population of cows within the first $2 \mathrm{yr}$ was not large enough to facilitate the selection of 3,000 females from the commercial tier, the program selected as large a number of females as was feasible. Of the 3,500 females born annually, 3,000 entered the commercial tier and the 500 elite females entered the elite tier based on the selection path of these animals (i.e., elite animals are born out of sires of sires and dams of sires matings; commercial females are born out of sires of cows and dams of cows matings). Within the commercial tier, 30 selected males were mated to the 3,000 females annually, with the resulting 3,000 female progeny considered as possible selection candidates for dams of sires and dams of dams (i.e., females could potentially become dams of elite tier animals, based on their genetic merit). Although the 1:1 ratio between cows and their resulting female offspring is not possible in practice, the authors did look at doubling the size of the commercial tier to 6,000 and found that it changed the results very little but did increase the computational time greatly; thus, it was decided to leave the commercial tier at 3,000 cows. Of the 30 selected males, the top 10 were chosen and mated to females within the elite tier. In elite matings, multiple ovulation and embryo transfer (MOET) was used such that each mating resulted in both a male and female offspring; thus, the elite matings resulted in 500 male and 500 female calves born annually (Figure $1)$. The bull calves were all potential selection candidates and were genotyped across all genomic schemes. Females remained as potential selection candidates from 2 to 6 yr of age. From the age of 3 onwards, a random third of the cows were culled annually. All matings were random where the parents of each offspring were sampled with replacement among the selected sires and dams. All animals that are selected and genotyped are used for the re-estimation of SNP effects annually.

\section{Genome}

In total, 30 chromosomes of equal length $(100 \mathrm{cM})$ were simulated in the genome, such that the total genome size was $3,000 \mathrm{cM}$. Bi-allelic markers were evenly distributed along the chromosomes at equal densities. The mutation rate was $10^{-2}$ per $\mathrm{cM}$ per generation across the 4,000 initial generations. The infinite sites mutation model was used, where each mutation gives rise to a bi-allelic SNP that occurred at a unique position. Per chromosome, 100 SNP with a minor allele frequency $(\mathbf{M A F})>5 \%$ were randomly selected, without replacement, to represent QTL. Among the remaining SNP within each chromosome, 500 with the highest MAF were chosen at random as genetic markers, resulting in a total of 15,000 markers and 3,000 QTL across the entire genome. For computational reasons, 


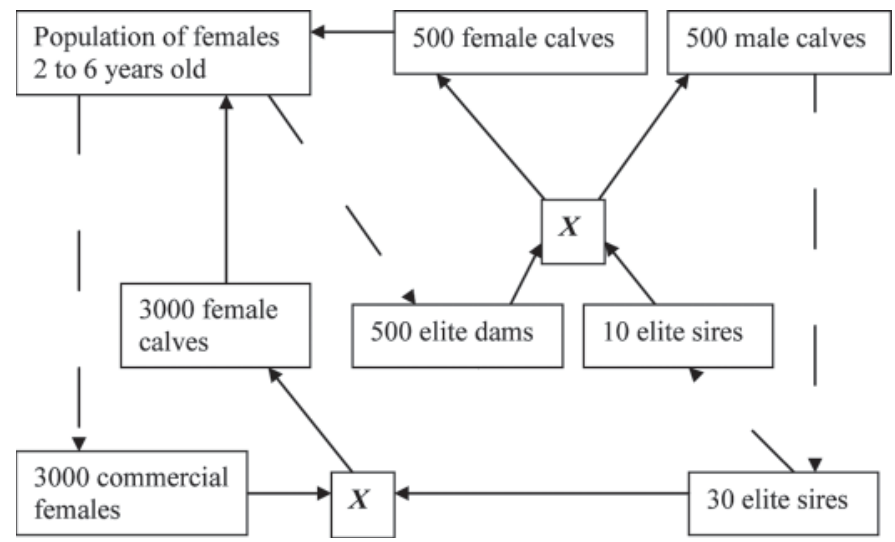

Figure 1. Population structure showing the 2 tiers (elite and commercial) and the various pathways that result in the selection (dashed line) of males and females and the eventual return and use ( $\mathbf{X}$ indicating the mating steps) of the proven sires to the 2 tiers.

the number of markers is kept quite low here, but the MAF of the markers is as high as possible. In addition, VanRaden et al. (2009) noted that decreasing the number of markers from 40,000 to 10,000 had little effect on the accuracy of genomic predictions.

The effects of the QTL were sampled from a gamma distribution with the shape parameter of 0.4 and a scale parameter of 1.66 (Meuwissen et al., 2001). The sign of the QTL effect was sampled to be either positive or negative with equal probability. Following sampling, the allelic effects of the QTL were standardized, resulting in a genetic variance of 1.0 per chromosome (i.e., the total genetic variance was 30; Sonesson and Meuwissen, 2009).

\section{Prediction of Phenotypes and True and Estimated Breeding Values}

Traditional EBV were estimated using BLUP methodology (Henderson, 1984), using information on the animal, if available, and all of its relatives. True breeding values (TBV), marker effects, and genome-wide breeding values were calculated as described in Sonesson and Meuwissen (2009).

The TBV of an animal was calculated as

$$
T B V_{i}=\sum_{j=1}^{3,000} \sum_{k=1}^{2} x_{i j k} g_{j k}
$$

where $x_{i j k}$ is the number of copies of the $k$ th allele that animal $i$ has at the $j$ th QTL position, and $g_{j k}$ is the effect of the $k$ th allele at the $j$ th position. The total genetic variance was standardized to 30 . The phenotypic values of an animal were simulated by adding to the TBV an error term sampled from a normal distribution
$N\left(0, \sigma_{e}^{2}\right)$, where $\sigma_{e}^{2}$ was 170 to simulate a trait heritability of 0.15 .

Marker effects were estimated using BLUP (Meuwissen et al., 2001), and re-estimated every year. The statistical model used to estimate the marker effects was

$$
y_{i}=\mu+\sum_{j=1}^{15,000} Z_{i j} a_{j}+e_{i}
$$

where $y_{i}$ is the record of test animal $i ; \mu$ is the overall mean; $Z_{i j}$ denotes the $j$ th marker genotype for animal $i$; with $-2 p_{j} / \sqrt{H_{j}}$, indicating that an individual is homozygous for the first allele, $\left(1-2 p_{j}\right) / \sqrt{H_{j}}$, and heterozygous and $\left(2-2 p_{j}\right) / \sqrt{H_{j}}$ homozygous for the second allele, where $H_{j}$ and $p_{j}$ are the marker heterozygosity and frequency, respectively; $a_{j}$ is the random effect of the $j$ th marker and $\operatorname{Var}\left(a_{j}\right)$ is assumed to be $30 / \mathrm{N}$ markers (total genetic variance was standardized to 30.0 ); and $e_{i}$ is a random residual whose variance was chosen such that the trait heritability was 0.15 .

Direct genomic values of the selection candidates were estimated by summing the effects of the markers:

$$
D G V_{i}=\sum_{j=1}^{15,000} Z_{i j} a_{j},
$$

where $D G V_{i}$ is the calculated DGV of animal $i ; a_{j}$ is the random effect of the $j$ th marker. The accuracy of selection (r) was calculated as the correlation between the TBV and EBV of the selection candidates.

\section{Breeding Schemes}

Several alternative breeding schemes (Table 1) were evaluated to quantify the potential use of female information within dairy breeding schemes, using population parameters representative of the Irish dairy cattle industry. Methods of selecting animals, number of animals genotyped, age of animals at selection, and number of animals used for the continual estimation of genetic marker effects were varied. Within each of the genomic breeding schemes, females were available as potential selection candidates from $2 \mathrm{yr}$ of age; within the traditional BLUP schemes, females were 3 yr of age before they were eligible as selection candidates. Across all schemes, females remained as selection candidates until 6 yr of age. Currently, within Ireland, bulls are culled for genetic defects and genetic merit for direct calving difficulty at $3 \mathrm{yr}$ of age and not released to widespread AI use until 6 yr of age. However, with 
increased confidence in DGV, the possibility exists of returning the bulls to widespread AI at 2 yr of age. With this in mind, males were available as selection candidates at either 2 or $3 \mathrm{yr}$ of age, depending on the genomic breeding scheme under investigation (Table 1); within the traditional BLUP scheme, males were available as selection candidates at 6 yr of age only. Across all schemes, males remained as selection candidates until 6 yr of age.

The primary breeding scheme examined the traditional method of selection based on breeding values estimated using BLUP procedures and the traditional additive genetic relationship matrix (trad_BLUP). Under this scheme, no animals were genotyped and animals were selected solely on their EBV. Every year, 1-yr-old males entered the progeny test and remained within this scheme until 6 yr of age and were only then available for selection; females were eligible as selection candidates at $3 \mathrm{yr}$.

The first breeding scheme to investigate the effect of using genomic selection is herein referred to as males_2yr. Here, genomic selection was used as a selection step for the identification of elite males only. Under the breeding scheme males_2yr, the 500 young bulls that were produced annually through the mating of the bull dams were all genotyped and were available as potential selection candidates, reaching a generation interval of 2 yr. No females were genotyped within this breeding scheme; females were chosen on their traditional EBV. Thereafter, the remaining 4 breeding schemes dealt with the effect of genotyping varying numbers of females (Table 1).

In the breeding scheme femaleT1_male3yr, all females within the elite tier (T1) were genotyped along with the males generated from the elite tier. Males were selected as potential selection candidates from $3 \mathrm{yr}$ onwards. In the breeding scheme femaleT1_male2yr, all progeny produced within the elite tier were genotyped and males were potential selection candidates at 2 yr of age. In the scheme, female_male3yr, the entire female population was genotyped along with the males generated from the elite tier; males were eligible as potential selection candidates at $3 \mathrm{yr}$ of age. In the final breeding scheme, female_male2yr, all females were genotyped (tier 1 and 2) and all males from the elite tier were eligible for selection from $2 \mathrm{yr}$ of age onwards.

Across all genomic selection schemes, males were chosen as selection candidates based on their DGV. Females, however, were selected based on EBV or DGV, depending on whether females were genotyped or not (Table 1). Male calves produced from the mating of elite cows within the elite tier (i.e., Tier 1) were genotyped, whereas the potential benefit of genotyping females

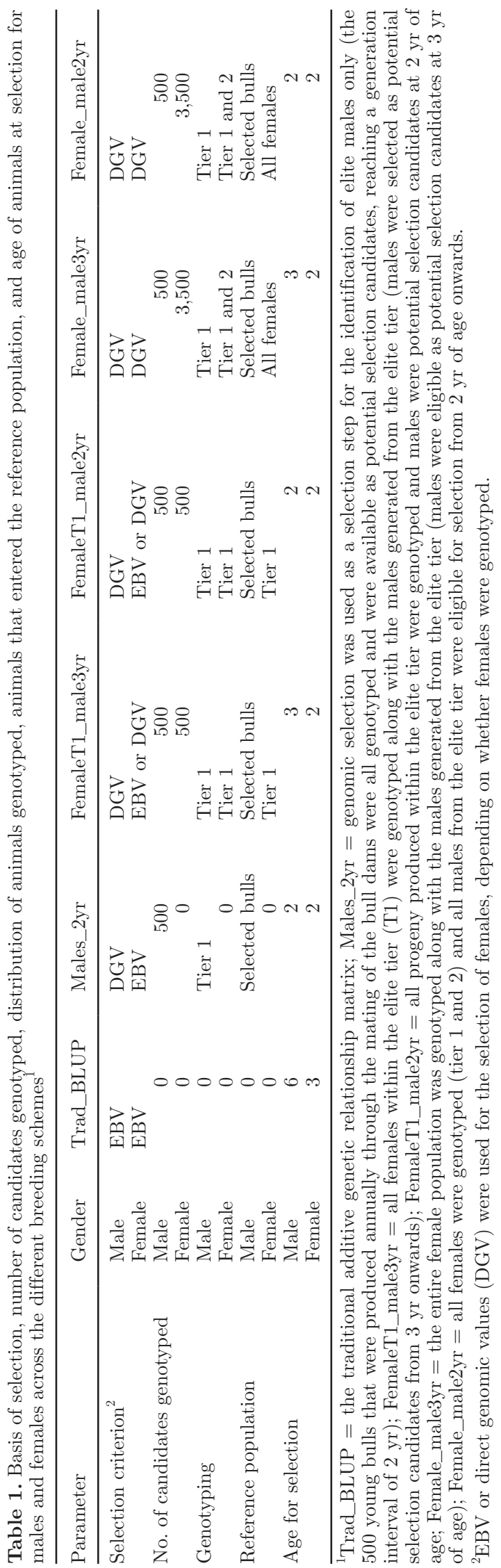


from both tiers was investigated. No structural progeny testing occurred in the genomic selection schemes.

\section{Statistics}

Selection schemes were run for $10 \mathrm{yr}$ (yr 1 to 10) and summary statistics presented for each of the schemes are based on 50 replicated simulations; only results from yr 7 to 10 are discussed. As explained previously, all animals within the base population were progeny tested and, therefore, these animals had high accuracy. These animals remained as potential selection candidates up to $6 \mathrm{yr}$ of age and, hence, yr 7 to 10 were used for comparing the various schemes when a steady state was approached: inbreeding started to accumulate and the true genomic breeding scheme was fully operational. Each of the different breeding schemes was compared on genetic gain $(\Delta \mathrm{G})$, accuracy of selection $(\mathrm{r})$, and rate of inbreeding per annum $(\Delta \mathrm{F})$, with inbreeding calculated based on pedigree of the animals (Meuwissen and Luo, 1992).

\section{RESULTS}

Irrespective of breeding scheme, genetic gain consistently increased across all years, although the rate of genetic gain varied among the different breeding schemes. Inbreeding levels followed a similar trend to genetic gain, increasing continuously across years (Figure 2). The accuracy of selection of males across all genomic schemes peaked in yr 1 because it was closely related to the initial training population, and decreased gradually thereafter, but approached a steady state from yr 7 onwards. The accuracy of selection of females followed a similar trend as the accuracy of males but the level of accuracy varied across the breeding schemes (Figure 2).

\section{Trad_BLUP}

Annual genetic gain for trad_BLUP averaged 0.17 genetic standard deviation units $\left(\sigma_{\mathrm{g}}\right)$ between yr 7 and 10; the corresponding rate of inbreeding was $0.14 \%$ per annum. The accuracy of the males averaged 0.22 in the final $3 \mathrm{yr}$ of the scheme (Figure 2). The generation interval for males was $6 \mathrm{yr}$. Results for females are not reported as results were generated for selected males only.

\section{Males_2yr}

The average annual rate of genetic gain for males_2yr was $0.34 \sigma_{\mathrm{g}}$ from yr 7 to 10 . The rate of inbreeding was, on average, $0.7 \%$ per annum from yr 7 to 10 (Figure 2).
The accuracy of all male candidates reached a plateau from yr 7 to 10 at approximately 0.77 . The accuracy of the females followed a similar trend to the males, with a considerable decrease in accuracy from 0.97 in yr 2 to 0.67 at yr 7 when steady state was reached (Figure 2 ). The average generation interval from yr 7 to 10 for males and females was 2.33 and $2.62 \mathrm{yr}$, respectively.

\section{FemaleT1_male3yr}

The average annual rate of genetic gain for femaleT1_male3yr was $0.33 \sigma_{\mathrm{g}}$ from yr 7 to 10 (Figure 2 ). The rate of inbreeding increased, on average, by $0.4 \%$ per annum across the same period. The accuracy of males remained relatively constant from yr 7 onwards at approximately 0.74 . The accuracy of females decreased from 0.68 to 0.61 from yr 7 to 10 ; many (i.e., tier 2 females) of the female selection candidates did not have DGV, but rather were selected on their traditional EBV. The average generation interval from yr 7 to 10 for males and females was 3.30 and $2.49 \mathrm{yr}$, respectively.

\section{FemaleT1_male2yr}

The average annual rate of genetic gain increased at a constant rate from yr 7 to 10 at an annual rate of 0.37 $\sigma_{\mathrm{g}}$. The rate of inbreeding rose steadily at an average rate of $0.4 \%$ per annum across yr 7 to 10 (Figure 2). The accuracy of the male candidates varied from 0.81 to 0.78 across yr 7 to 10 . The accuracy of the females averaged 0.81 across all $10 \mathrm{yr}$, with a lower accuracy recorded in the final years varying from 0.66 to 0.73 in yr 7 to 10 . The generation interval averaged across yr 7 to 10 was 2.28 and 2.38 yr for males and females, respectively.

\section{Female_male3yr}

The average annual rate of genetic gain for female_ male3yr increased at a constant rate across yr 7 to 10 at an annual rate of $0.42 \sigma_{\mathrm{g}}$. The rate of inbreeding increased steadily across the same years at an average rate of $0.4 \%$ per annum (Figure 2). The accuracy associated with the male candidates decreased in the first $6 \mathrm{yr}$ but reached a steady state thereafter ( 0.78 to 0.81 ; yr 7 to 10). The accuracy of the females averaged 0.82 in the final $3 \mathrm{yr}$ of the scheme (Figure 2). The generation interval associated with the males and females was 3.34 and $2.23 \mathrm{yr}$, respectively.

\section{Female_male2yr}

Genetic gain increased continuously across yr 7 to 10 at an average rate of $0.49 \sigma_{\mathrm{g}}$ per annum. The rate 
a)

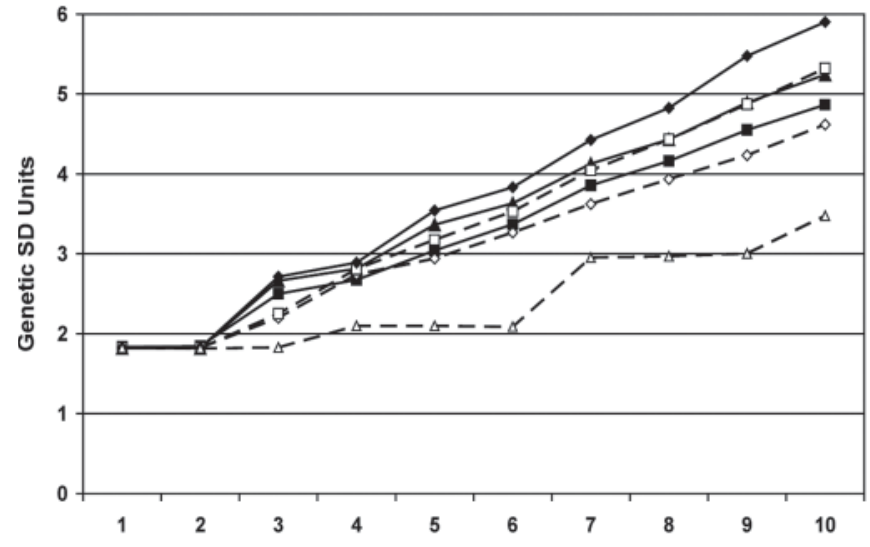

b)

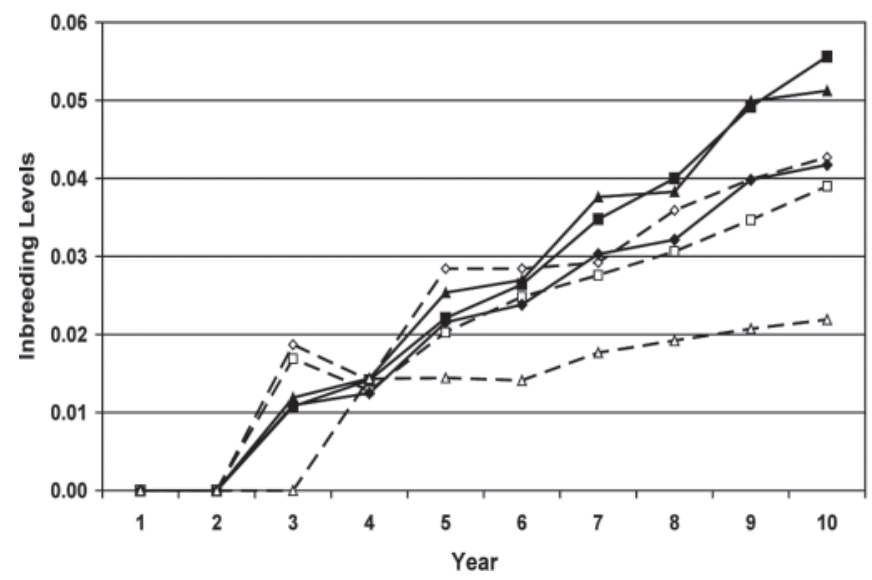

c)

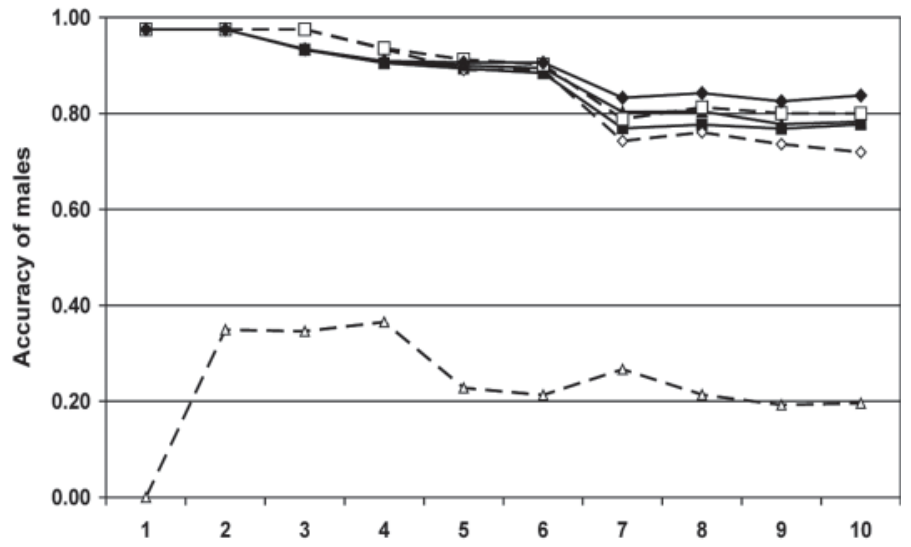

d)

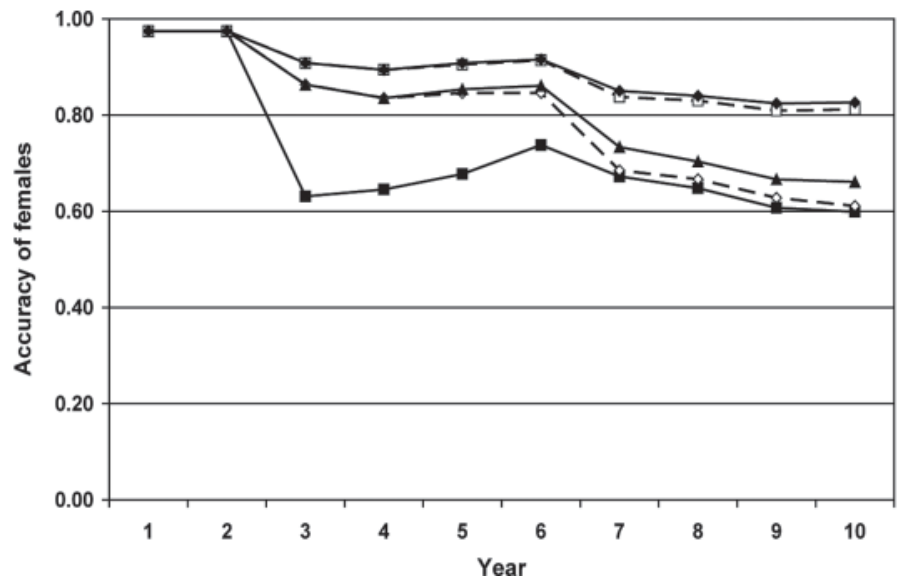

Figure 2. Change in (a) genetic level, (b) rate of inbreeding, (c) accuracy of males, and (d) accuracy of females from yr 1 to 10 for the traditional additive genetic relationship matrix [Trad_BLUP $(-\Delta-)$ ], the scheme in which genomic selection was used as a selection step for the identification of elite males only [males_2yr (-ם-); the 500 young bulls that were produced annually through the mating of the bull dams were all genotyped and were available as potential selection candidates, reaching a generation interval of $2 \mathrm{yr}$ ], the scheme in which all females within the elite tier (T1) were genotyped along with the males generated from the elite tier [femaleT1_male3yr (- $\diamond-)$; males were selected as potential selection candidates from $3 \mathrm{yr}$ onwards], the scheme in which all progeny produced within the elite tier were genotyped and males were potential

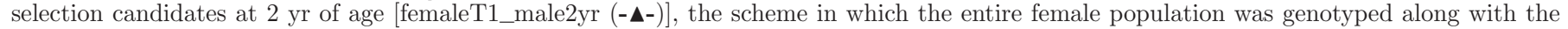
males generated from the elite tier [female_male3yr (- $\square$-); males were eligible as potential selection candidates at 3 yr of age], and the scheme in which all females were genotyped (tier 1 and 2) and all males from the elite tier were eligible for selection from 2 yr of age onwards [female_. male2yr (- -)]. Mean standard errors for genetic level from yr 1 to 10 were 0.07, 0.13, 0.08, 0.11, 0.09, and 0.11 for Trad_BLUP, males_2yr, femaleT1_male3yr, femaleT1_male2yr, female_male3yr, and female_male2yr, respectively.

of inbreeding recorded across the same period for female_male2yr was $0.4 \%$ (Figure 2). The accuracy of the males decreased from yr 1 to 6 but remained constant thereafter, varying from 0.82 to 0.84 (yr 7 to 10). The accuracy of the females averaged 0.83 across yr 7 to 10 . The generation interval of both the males and females was 2.22 and $2.21 \mathrm{yr}$, respectively.

\section{DISCUSSION}

With increasing interest in the incorporation of genomic selection into dairy breeding programs worldwide, research needs to focus on the optimum use of genomic selection in national breeding programs. Con- tinuous re-estimation of the marker effects, through the establishment of a population with accurate phenotypes, is needed for the successful implementation of genomic selection (Habier et al., 2007). The lack of a continued emergence of new proven sires annually with accurate phenotypes, especially in small breeding programs, necessitates an evaluation of other potential sources of data for continual re-estimation of marker effects. The objective of this paper was 2-fold: to evaluate the potential use of female phenotypes and genotypes in the estimation of marker effects and the effect female genomic selection has on genetic gain. Schaeffer (2006) reported a potential doubling of genetic gain with genomic selection using population parameters 
representative of the Canadian dairy cattle population. The present study shows that the inclusion of females in genomic selection breeding programs can accelerate genetic gain almost 3-fold through increased accuracy of selection and decrease of generation intervals, while maintaining a reasonable rate of inbreeding. The accuracy of the selected males was increased by $73 \%$ in the final $3 \mathrm{yr}$ of the genomic schemes compared with the traditional BLUP scheme. It is, therefore, recommended that the Irish dairy industry commence genotyping females on a large-scale basis.

For computational reasons, all schemes were run for a relatively short period, $10 \mathrm{yr}$, and had only 50 replicates per scheme. However, from yr 7 onwards, the genomic breeding schemes became truly operational and the year-to-year variation between schemes was relatively small among the schemes.

\section{Genetic Gain and Accuracy of Selection}

Results from this simulation have shown that the inclusion of female phenotypic and genomic information can lead to an almost 3-fold increase in the rate of genetic gain ( 0.17 to $0.49 \sigma_{\mathrm{g}}$ per annum) in the final 3 yr of the schemes when compared with the traditional BLUP scheme. Previous simulation studies have shown an annual rate of genetic gain in genomic selection schemes of between 49 and 231\% (Buch et al., 2010; Lillehammer et al., 2010; Pryce et al., 2010) relative to traditional BLUP breeding programs.

The accuracy of using genomic selection for both males and females in the present study were consistent with accuracies reported elsewhere for males (Schaeffer, 2006; König et al., 2009; Nielsen et al., 2009) and females (Schaeffer, 2006). Daetwyler et al. (2007) concluded that the increase in accuracy associated with genomic selection breeding programs, compared with traditional BLUP schemes, resulted in lower between-family variance and increased selective pressure on the Mendelian sampling term. The latter is expected to decrease the rate of inbreeding per generation when compared with traditional BLUP (Daetwyler et al., 2007), but in the present study the generation intervals were simultaneously decreased by a factor of 2 to 3 , which increased the annual rates of inbreeding. It is expected that, over time, the accuracy of genomic breeding values will decrease because of recombination between markers and QTL (Habier et al., 2007). However, within the present study, SNP effects were re-estimated annually and accuracies reached a plateau for all genomic schemes from yr 7 onwards. Studies have shown that accuracy of selection is dependent on a multitude of factors, including number of generations with phenotypic and genomic information (Habier et al., 2007; Muir, 2007; Sonesson and Meuwissen, 2009), marker density (Solberg et al., 2008; Luan et al., 2009; Nielsen et al., 2009), and heritability of the trait under selection (Hayes et al., 2009; Luan et al., 2009; Calus, 2010). Within the genomic schemes investigated in the present study, the accuracy for both males and females did not decrease after yr 7 (Figure 2) because of the contribution of the EBV from the elite sires to the re-estimation of the SNP effects. However, this result depends on the number of sires selected; when the number of sires selected for mating with both the elite and commercial tier was decreased to 20 annually, the accuracy of both the males and females decreased over time (results not shown).

Previous studies have shown that to maintain a high level of accuracy across generations, animals used for re-estimation of the marker effects should comprise a diverse range of phenotypes and genotypes (Calus, 2010). Within the present study, greater accuracies for both male and females were associated with the schemes where larger numbers of phenotyped animals were genotyped (i.e., female_male3yr and female_male2yr). The greater accuracies in these schemes may partly be because of the increase in the number of records used to re-estimate the marker effects across the various schemes (i.e., the total reference population after 10 yr for males_2yr and females_male2yr consisted of 8,000 and 39,000, respectively). Increased genetic gains were also due to the fact that females were genotyped, thus facilitating higher selection intensity and a shorter generation interval in the dams to breed bull's pathway. Sonesson and Meuwissen (2009) also speculated that under a genomic selection breeding scheme, the accuracy of selection will remain high across generations as the favorable alleles may become more frequent within the population and are, thus, more accurately estimated.

Within Irish commercial dairy herds, preferential treatment of females is not practiced; therefore, the effect of preferential treatment in the female population, particularly in the elite tier, was ignored in this study. However, the influence of preferential treatment on traditional cow EBV and, subsequently, the estimation of SNP effects are an important issue in a large number of countries. Although potential methods to account for preferential treatment have been suggested, none are optimal and further research is necessary to determine how to optimally minimize the influence of preferential treatment.

\section{Generation Interval}

With increasing farmer confidence in genomic selection, the age at which young bulls are released for widespread AI use is likely to decrease. Harris et al. 
(2008) concluded that bulls should not be available for widespread use until $2 \mathrm{yr}$ of age when bulls are checked for congenital defects based on progeny born. Within the present study, the availability of bulls for widespread use at $2 \mathrm{yr}$ of age not only leads to a decrease in the generation interval of the males, but also leads to an increase in genetic gain and the accuracy of selection for both male and female candidates. König et al. (2009) reported that a decrease in generation interval, coupled with an increase in accuracies, can increase the economic benefits of genomic selection breeding programs by a factor of 2 for the overall dairy population.

\section{Inbreeding}

Currently, the annual rate of increase in inbreeding within the Irish Holstein-Friesian dairy population is $0.10 \%$ (Mc Parland et al., 2007). A rate of increase in inbreeding of $\leq 1 \%$ per generation is considered acceptable (Goddard, 1992). Although rates of inbreeding per annum calculated within the present study are higher than the levels suggested by Goddard (1992) and calculated by Mc Parland et al. (2007), it should be remembered that this simulation study was smaller than the actual Irish dairy cattle population. If we compare our inbreeding results on a per-generation basis, we see that slightly higher rates are still reported for the genomic schemes compared with the traditional BLUP scheme. A previous simulation study (Daetwyler et al., 2007) reported the opposite trend in inbreeding per generation, with greater inbreeding levels associated with traditional BLUP schemes compared with genomic selection schemes, using a similar heritability estimate to the present study $\left(\mathrm{h}^{2}=0.15\right)$. However, within our study, bulls in the traditional BLUP scheme were not available for AI use until $6 \mathrm{yr}$ of age, which had 2 effects: (1) the trad_BLUP scheme had a longer generation interval compared with the genomic schemes and (2) the trad_BLUP scheme had highly accurately estimated within-family effects because the progeny testing and genomic selection could not improve these. Koenig and Simianer (2006) stated that the long-term control of inbreeding requires consideration of genetic relationship between young bulls entering AI breeding programs; emphasis, therefore, may shift to the cow side of the pedigree and, hence, decrease the problem caused by too few sires of replacements (Schaeffer, 2006). The use of genomic selection facilitates the identification of the least related animals more accurately than the traditional relationship matrix (Sonesson et al., 2010). Results from the present study support this theory, with lower rates of inbreeding associated with the genotyping of a large number of females. Ideally, optimum contribution selection should to be applied to constrain the rate of inbreeding (Meuwissen and Sonesson, 1998).

The use of the traditional relationship matrix for the estimation of inbreeding rates using genomic selection is limited compared with genomic relationship matrices. Under the traditional relationship method, the relationship between an animals and its grandsire is assumed to be 0.25; however, VanRaden (2007) has shown that genomic relationship matrices can estimate the expected fraction of genes identical by descent, the actual fraction of DNA shared, and thus, can assess the relationship between individuals to a higher degree of accuracy.

\section{Cost Effectiveness}

Although additional costs would be associated with the genotyping of females compared with both the trad_BLUP and males_2yr schemes, König et al. (2009) showed in their study that the costs associated with the genotyping of a large number of animals $(\mathrm{n}=$ 250 animals) was offset by the greater monetary genetic gain associated with the implementation of genomic breeding schemes. König et al. (2009) also reported that the increased cost associated with genotyping of animals was negligible compared with the costs of herd book registration, milk recording, and progeny testing of young bulls.

Weller (1994) suggested that the long-term profitability of a breeding program is a function of the expected costs and returns of the breeding program as well as an appropriate discount rate and profit time horizon. The question remains, however, which cost-benefit analyses should be undertaken: from an individual breeding company perspective or for the country as a whole, with or without market share in an export market. The genetic standard deviation of the Irish total merit index for dairy cattle, the Economic Breeding Index (EBI) is $€ 69$ (Berry et al., 2007). Given the potential genetic gain of $0.49 \sigma_{g}$ units achievable annually between yr 7 and 10 in female_male2yr, this has the potential to increase the value per cow by $€ 25.36$ per annum across the $4 \mathrm{yr}$. Across the Irish dairy cow population of 1.15 million dairy cows, the potential increase in profitability and, therefore, return on investment, is large.

Schaeffer (2006) reported that the use of genomic selection breeding schemes decreases the industry need for progeny testing schemes and can potentially decrease the costs of proving bulls by up to $92 \%$.

Another possible option for decreasing the costs associated with genotyping a large number of females is to use a less dense set of markers and impute up to 54,000 SNP (Weigel et al., 2010; Zhang and Druet, 2010). High imputation accuracies are achievable and the lower 
associated costs may act as incentives for farmers to genomically select their females.

\section{Implications}

The results of the present study have several implications for national breeding schemes. Although an investment in genotyping a large population of animals is required, these costs can be offset by the greater genetic gains achievable through the increased accuracy of selection associated with genomic selection. Also, with the continual decrease in the cost of genotyping animals, coupled with imputation of genotypes, a scenario where the entire female population is genotyped may become a reality in the near future. The additional costs of genotyping the female population may be shouldered by the entire industry as the undertaking will have benefits, not only for the individual farmer, but to the entire breeding industry.

Within the present study, we found that genomic selection can increase the accuracy of selection of young males, after $7 \mathrm{yr}$, from 0.22 for the traditional BLUP scheme to between 0.74 and 0.83 using genomic breeding schemes. The accuracy of the female population also followed a similar trend, with the genomic schemes yielding an accuracy of between 0.67 and 0.85 after 7 yr. Fluctuations in the estimates of genetic merit for young animals have a subsequent effect on farmer confidence in the genetic evaluations system.

Under the assumption that a high accuracy progeny testing scheme is in place, genomic breeding programs have also been shown to decrease the need for progeny testing. We showed that data from individual cows could be used instead to generate phenotypic training data as long as animals closely related to the selection candidates are used for estimating DGV.

\section{ACKNOWLEDGMENTS}

The authors acknowledge the extensive input and direction of Donagh Berry, Teagasc, Moorepark, Ireland.

\section{REFERENCES}

Berry, D. P., J. F. Kearney, and B. L. Harris. 2009. Genomic selection in Ireland. In Proc. Interbull International Workshop, Uppsala, Sweden. Bulletin No. 39, 2009. ISSN 1011-6079. Interbull, Uppsala, Sweden.

Berry, D. P., L. Shalloo, A. R. Cromie, V. Olori, R. F. Veerkamp, P. Dillon, P. R. Amer, R. D. Evans, J. F. Kearney, and B. Wickham. 2007. The economic breeding index: A generation on. Technical report to the Irish Cattle Breeding Federation.

Buch, L. H., M. K. Sorensen, J. Lassen, P. Berg, and A. C. Sorensen. 2010. Dairy Cattle Breeding Schemes with or without genomic selection and progeny testing. In Proc. 9th World Congr. Genet. Appl. Livest. Prod. Leipzig, Germany. Gesellschaft fur Tier- zuchtwissenschaften e. V. Leipzig, Germany, CD- Rom, Comm. 0418.

Calus, M. P. L. 2010. Genomic breeding value prediction: Methods and procedures. Animal 4:157-164.

Daetwyler, H. D., B. Villanueva, P. Bijma, and J. A. Woolliams. 2007. Inbreeding in genome-wide selection. J. Anim. Breed. Genet. 124:369-376.

Fisher, R. A. 1930. The Genetical Theory of Natural Selection. Clarendon Press, Oxford, UK.

Goddard, M. E. 1992. Optimal effective population size for the global population of black and white dairy cattle. J. Dairy Sci. 75:29022911.

Habier, D., R. L. Fernando, and J. C. M. Dekkers. 2007. The impact of genetic relationship information on genome-assisted breeding values. Genetics 177:2389-2397.

Harris, B. L., and D. L. Johnson. 2010. Genomic predictions for New Zealand dairy bulls and integration with national genetic evaluation. J. Dairy Sci. 93:1243-1252.

Harris, B. L., D. L. Johnson, and R. J. Spelman. 2008. Genomic selection in New Zealand and the implications for national genetic evaluation. Pages 325-330 in Proc. 36th ICAR Biennial Session, Niagara Falls, NY. ICAR Technical Series 2009, No. 13. International Committee for Animal Recording (ICAR), Rome, Italy.

Hayes, B. J., P. J. Bowman, A. J. Chamberlain, and M. E. Goddard. 2009. Invited review: Genomic selection in dairy cattle: Progress and challenges. J. Dairy Sci. 92:433-443.

Henderson, C. R. 1984 Applications of Linear Models in Animal Breeding. Guelph Univ. Press, Guelph, ON, Canada.

Koenig, S., and H. Simianer. 2006. Approaches to the management of inbreeding and relationship in the German Holstein dairy cattle population. Livest. Sci. 103:40-53.

König, S., H. Simianer, and A. Willam. 2009. Economic evaluation of genomic breeding programs. J. Dairy Sci. 92:382-391.

Lillehammer, M., T. H. E. Meuwissen, and A. K. Sonesson. 2010. Effects of alternative genomic selection breeding schemes on genetic gain in dairy cattle. In Proc. 9th World Congr. Genet. Appl. Livest. Prod. Leipzig, Germany. Gesellschaft fur Tierzuchtwissenschaften e. V. Leipzig, Germany, CD- Rom, Comm. 0130.

Luan, T., J. A. Woolliams, S. Lien, M. Kent, M. Svendsen, and T. H. E. Meuwissen. 2009. The accuracy of genomic selection in Norwegian Red cattle assessed by cross validation. Genetics 183:1119-1126.

Mc Parland, S., J. F. Kearney, M. Rath, and D. P. Berry. 2007. Inbreeding trends and pedigree analysis of Irish dairy and beef cattle populations. J. Anim. Sci. 85:322-331.

Meuwissen, T. H., and A. K. Sonesson. 1998. Maximizing the response of selection with a predefined rate of inbreeding: Overlapping generations. J. Anim. Sci. 76:2575-2583.

Meuwissen, T. H. E., B. J. Hayes, and M. E. Goddard. 2001. Prediction of total genetic value using genome-wide dense marker maps. Genetics 157:1819-1829.

Meuwissen, T. H. E., and Z. Luo. 1992. Computing inbreeding coefficients in large population. Genet. Sel. Evol. 24:305-313.

Muir, W. M. 2007. Comparison of genomic and traditional BLUPestimated breeding value accuracy and selection response under alternative trait and genomic parameters. J. Anim. Breed. Genet. 124:342-355.

Nielsen, H. M., A. K. Sonesson, H. Yazdi, and T. H. E. Meuwissen. 2009. Comparison of accuracy of genome-wide and BLUP breeding value estimates in sib based aquaculture breeding schemes. Aquaculture 289:259-264.

Pryce, J. E., M. E. Goddard, H. W. Raadsma, and B. J. Hayes. 2010. Deterministic models of breeding scheme designs that incorporate genomic selection. J. Dairy Sci. 93:5455-5466.

Schaeffer, L. R. 2006. Strategy for applying genome-wide selection in dairy cattle. J. Anim. Breed. Genet. 123:218-223.

Sellner, E. M., J. W. Kim, M. C. McClure, K. H. Taylor, R. D. Schnabel, and T. F. Taylor. 2007. Board-invited review: Applications of genomic information in livestock. J. Anim. Sci. 85:3148-3158.

Solberg, T. R., A. K. Sonesson, J. A. Woolliams, and T. H. E. Meuwissen. 2008. Genomic selection using different marker types and densities. J. Anim. Sci. 86:2447-2454. 
Sonesson, A. K., and T. H. E. Meuwissen. 2009. Testing strategies for genomic selection in aquaculture breeding programs. Genet. Sel. Evol. 41:37.

Sonesson, A. K., J. A. Woolliams, and T. H. E. Meuwissen. 2010 Maximising genetic gain whilst controlling rates of genomic inbreeding using genomic optimum contribution selection. In Proc. 9th World Congr. Genet. Appl. Livest. Prod. Leipzig, Germany. Gesellschaft fur Tierzuchtwissenschaften e. V. Leipzig, Germany, CD- Rom, Comm.

VanRaden, P. M. 2007. Genomic measures of relationship and inbreeding. Pages 33-36 in Proc. Interbull Annual Meeting. Interbull, Uppsala, Sweden

VanRaden, P. M., C. P. Van Tassell, G. R. Wiggans, T. S. Sonstegard, R. D. Schnabel, J. F. Taylor, and F. S. Schenkel. 2009. Invited review: Reliability of genomic predictions for North American Holstein bulls. J. Dairy Sci. 92:16-24.

Weigel, K. A., C. P. Van Tassell, J. R. O'Connell, P. M. VanRaden, and G. R. Wiggans. 2010. Prediction of unobserved single nucleotide polymorphism genotypes of Jersey cattle using panels and population-based imputation algorithms. J. Dairy Sci. 93:22292238 .

Weller, J. I. 1994. Economic Aspects of Animal Breeding. Chapman \& Hall, London, UK.

Wright, S. 1931. Evolution in Mendelian populations. Genetics 16:97159.

Zhang, Z., and T. Druet. 2010. Marker imputation with low-density marker panels in Dutch Holstein cattle. J. Dairy Sci. 93:54875494 . 